\title{
HIGH-ORDER ANALYTICAL SOLUTIONS OF HILL'S EQUATIONS
}

\author{
G. GÓMEZ and M. MARCOTE \\ Departament Matemàtica Aplicada i Anàlisi, Universitat de Barcelona, Gran Via 585 , \\ 08007 Barcelona, Spain, e-mails: \{gerard, marcote\}@maia.ub.es
}

(Received: 12 July 2005; accepted: 7 November 2005)

\begin{abstract}
The purpose of this paper is the semi-analytical computation of the bounded orbits of Hill's equations, describing the relative motion of two particles in their Keplerian motion around a central body. We have considered the case in which one of the particles moves along a circular reference orbit. The solutions obtained are the generalisation of the usual periodic orbits obtained for the linearised equations and also of the third-order solution computed by D.L. Richardson and J.W. Mitchell (2003). With the algorithm presented, those orbits can be computed in a fast and efficient way up to a high-order in the in-plane and out-of-plane amplitudes.
\end{abstract}

Key words: Hill's equations, Lindstedt-Poincaré series

\section{Introduction}

Hill's, or Clohessy-Wiltshire, equations describe the relative motion of two point-mass bodies under the gravitational influence of a point-mass central body. The bounded solutions of these equations are useful for several problems, such as the determination of formation flight suitable trajectories or the description of rendezvous manoeuvres. Usually, Hill's equations are considered in their linear approximation with one of the bodies moving along a circular orbit. For these linear equations is easy to get a bi-parametric family of periodic orbits. In a recent paper by Richardson and Mitchell (2003) the linear approximation is extended to a third order solution in the two parameters of the family (the in-plane and the out-of-plane amplitudes). In the present paper, using also the Lindstedt-Poincare procedure for their determination, we extend the computations of Richardson and Mitchell to an arbitrary order, providing in this way more accurate results.

\section{The Model}

As it has already been said, Hill's equations can be used to describe the relative motion of two spacecrafts subjected to the gravitational influence 
of a point-mass central body. We will assume that one of the spacecrafts, the leader, moves in a circular reference orbit of radius $r_{l}$ about the central body and that the second spacecraft, the follower, moves close to the leader, at a distance $\delta r$ from it. If $\mathbf{r}_{l}$ and $\mathbf{r}_{f}$ denote the inertial positions of the leader and the follower, respectively, with respect to the central body, then $\mathbf{r}_{f}=\mathbf{r}_{l}+\delta \mathbf{r}$ (see Figure 1).

For the description of the motion, is useful to introduce Hill's non-inertial reference system, $\mathbf{e}_{r}, \mathbf{e}_{\theta}, \mathbf{e}_{c}$, centred at the leader and defined by

$$
\mathbf{e}_{r}=\frac{\mathbf{r}_{l}}{r_{l}}, \quad \mathbf{e}_{c}=\frac{\mathbf{r}_{l} \wedge \mathbf{r}_{l}^{\prime}}{\left\|\mathbf{r}_{l} \wedge \mathbf{r}_{l}^{\prime}\right\|}, \quad \mathbf{e}_{\theta}=-\mathbf{e}_{r} \wedge \mathbf{e}_{c}
$$

where $r_{l}=\left\|\mathbf{r}_{l}\right\|$ and the prime denotes the time-derivative. To obtain the relative equations of motion in Hill's coordinates, we write the follower satellite position vector as

$$
\mathbf{r}_{f}=\mathbf{r}_{l}+\delta \mathbf{r}=\left(X+r_{l}\right) \mathbf{e}_{r}+Y \mathbf{e}_{\theta}+Z \mathbf{e}_{c} .
$$

Using that $\mathbf{e}_{r}^{\prime}=n \mathbf{e}_{\theta}, \mathbf{e}_{\theta}^{\prime}=-n \mathbf{e}_{r}$, where $n$ is the mean motion of the leader on its circular orbit, we get that the follower acceleration is given by

$$
\mathbf{r}_{f}^{\prime \prime}=\left(X^{\prime \prime}-2 n Y^{\prime}-n^{2}\left(X+r_{l}\right)\right) \mathbf{e}_{r}+\left(Y^{\prime \prime}+2 n X^{\prime}-n^{2} Y\right) \mathbf{e}_{\theta}+Z^{\prime \prime} \mathbf{e}_{c} .
$$

The follower equations of motion are given by

$$
\mathbf{r}_{f}^{\prime \prime}=-\frac{\mu}{r_{f}^{3}} \mathbf{r}_{f}=-\frac{\mu}{r_{f}^{3}}\left(\begin{array}{c}
X+r_{l} \\
Y \\
Z
\end{array}\right),
$$

with

$$
r_{f}=\sqrt{\left(X+r_{l}\right)^{2}+Y^{2}+Z^{2}}
$$

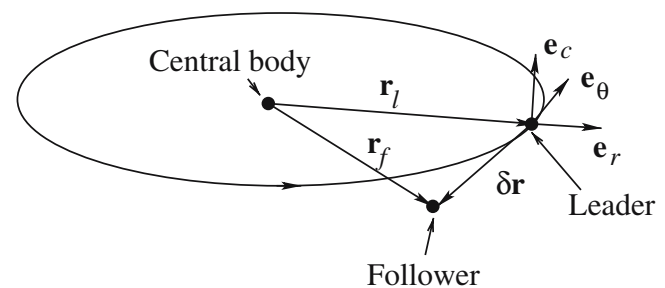

Figure 1. Reference system for the description of the relative motion of the follower spacecraft with respect to the leader. 
Equating (1) and (2) we get the nonlinear relative equations of motion

$$
\begin{aligned}
X^{\prime \prime}-2 n Y^{\prime}-n^{2}\left(X+r_{l}\right) & =-\frac{\mu}{r_{f}^{3}}\left(X+r_{l}\right), \\
Y^{\prime \prime}+2 n X^{\prime}-n^{2} Y & =-\frac{\mu}{r_{f}^{3}} Y, \\
Z^{\prime \prime} & =-\frac{\mu}{r_{f}^{3}} Z .
\end{aligned}
$$

Introducing dimensionless coordinates $x, y, z$, dimensionless time $t$ via

$$
X=r_{l} x, \quad Y=r_{l} y, \quad Z=r_{l} z, \quad \mathrm{~d} t=n \mathrm{~d} \tau,
$$

and using that $\mu=n^{2} r_{l}^{3}$, the relative equations of motion can be written as

$$
\begin{aligned}
\ddot{x}-2 \dot{y} & =\Omega_{x}, \\
\ddot{y}+2 \dot{x} & =\Omega_{y}, \\
\ddot{z} & =\Omega_{z},
\end{aligned}
$$

where

$$
\Omega(x, y, z)=\frac{(x+1)^{2}+y^{2}}{2}+\frac{1}{\sqrt{(x+1)^{2}+y^{2}+z^{2}}} .
$$

In order to expand the nonlinear terms of (3), we use that

$$
\frac{1}{\sqrt{(x-A)^{2}+(y-B)^{2}+(z-C)^{2}}}=\frac{1}{D} \sum_{n \geqslant 0}\left(\frac{\rho}{D}\right)^{n} P_{n}\left(\frac{A x+B y+C z}{D \rho}\right),
$$

where $D^{2}=A^{2}+B^{2}+C^{2}, \rho^{2}=x^{2}+y^{2}+z^{2}$ and $P_{n}$ is the Legendre polynomial of degree $n$ (see Abramowitz and Stegun, 1972). In this way, and since in our case $A=-1, B=C=0$, Equations (3) become

$$
\begin{aligned}
\ddot{x}-2 \dot{y}-3 x & =\frac{\partial}{\partial x} \sum_{n \geqslant 3} \rho^{n} P_{n}\left(-\frac{x}{\rho}\right), \\
\ddot{y}+2 \dot{x} & =\frac{\partial}{\partial y} \sum_{n \geqslant 3} \rho^{n} P_{n}\left(-\frac{x}{\rho}\right), \\
\ddot{z}+z & =\frac{\partial}{\partial z} \sum_{n \geqslant 3} \rho^{n} P_{n}\left(-\frac{x}{\rho}\right) .
\end{aligned}
$$

The above equations are the starting point of our computations, which follow closely those done for the determination of Lissajous orbits around 
the collinear libration points of the Restricted Three Body Problem (RTBP) (see Gómez et al. 2001; Masdemont, 2005). This is a very convenient formulation of the equations of motion, from the point of view of algebraic manipulation, and was introduce for the first time in Richardson (1980) for the computation of halo orbits around the collinear libration points of the RTBP. The equations for the problem under consideration can be also obtained from the ones of the RTBP just setting the $c_{n}$ constants that appear in these equations equal to one.

It is convenient to write down the right-hand side of (4) using functions which can be computed (and stored) in a fast and easy way. This is done by introducing the functions

$$
T_{n}(x, y, z)=\rho^{n} P_{n}\left(-\frac{x}{\rho}\right), \quad R_{n-1}(x, y, z)=\frac{1}{y} \frac{\partial T_{n+1}}{\partial y} .
$$

Is easy to verify that $T_{n}$ and $R_{n}$ are polynomials of degree $n$ satisfying

$$
R_{n-1}=\frac{1}{z} \frac{\partial T_{n+1}}{\partial z}, \quad \frac{\partial T_{n+1}}{\partial x}=-(n+1) T_{n} .
$$

Using these relations, Equations (4) become

$$
\begin{gathered}
\ddot{x}-2 \dot{y}-3 x=-\sum_{n \geqslant 2}(n+1) T_{n}, \\
\ddot{y}+2 \dot{x}=y \sum_{n \geqslant 2} R_{n-1}, \\
\ddot{z}+z=z \sum_{n \geqslant 2} R_{n-1},
\end{gathered}
$$

where $T_{n}$ and $R_{n}$ can be obtained using the recurrences that follow from the properties of the Legendre polynomials:

$$
\begin{aligned}
& T_{n}(x, y, z)=\frac{1-2 n}{n} x T_{n-1}-\frac{n-1}{n} \rho^{2} T_{n-2}, \\
& R_{n}(x, y, z)=-\frac{2 n+3}{n+2} x R_{n-1}+\frac{2 n+2}{n+2} T_{n}-\frac{n+1}{n+2} \rho^{2} R_{n-2},
\end{aligned}
$$

starting with: $T_{0}=1, T_{1}=-x, R_{0}=-1, R_{1}=3 x$.

\section{The Lindstedt-Poincaré Procedure}

If we remove from (5) the nonlinear right-hand side terms, the bounded solutions of the linear system obtained are $2 \pi$-periodic and can be written 
as

$$
\begin{aligned}
& x=\alpha \cos \left(t+\phi_{1}\right), \\
& y=-2 \alpha \sin \left(t+\phi_{1}\right), \\
& z=\beta \cos \left(t+\phi_{2}\right) .
\end{aligned}
$$

The parameters $\alpha, \beta$ are the in-plane and out-of-plane amplitudes of the orbit and $\phi_{1}, \phi_{2}$ the phases. When the nonlinear terms of (5) are considered, one can look for formal series solutions expanded in powers of the amplitudes $\alpha$ and $\beta$, having (6) as first order terms, of the type

$$
\left\{\begin{array}{l}
x \\
y \\
z
\end{array}\right\}=\sum_{i+j=1}^{\infty}\left(\sum_{|k| \leqslant i,|m| \leqslant j}\left\{\begin{array}{l}
x \\
y \\
z
\end{array}\right\}_{i j k m}\left\{\begin{array}{c}
\cos \\
\sin \\
\cos
\end{array}\right\}\left(k \theta_{1}+m \theta_{2}\right)\right) \alpha^{i} \beta^{j},
$$

where $\theta_{1}=\omega t+\phi_{1}, \theta_{2}=\omega t+\phi_{2}$. In order to avoid secular behaviours due to the nonlinear terms, the frequency $\omega$ cannot be kept equal to 1 and must be expanded also in powers of the amplitudes

$$
\omega=1+\sum_{i+j \geqslant 1} \omega_{i j} \alpha^{i} \beta^{j}
$$

The goal is to recurrently compute the coefficients $x_{i j k m}, y_{i j k m}, z_{i j k m}$ and $\omega_{i j}$ up to an arbitrary finite order $N$, this is: for all the values of $i$ and $j$ such that $1 \leqslant i+j \leqslant N$.

It must be remarked that $x$ and $z$ have been both written as a cosinus series and $y$ as a sinus one. This can be done due to the symmetries of the differential equations of the problem and to the selected expression for the solution of the linear part. One can see also that if $j$ is odd, the coefficients $x_{i j k m}$ and $y_{i j k m}$ are zero and if $j$ is even, then the coefficients $z_{i j k m}$ are zero. As is usual in the Lindstedt-Poincare method, we only use coefficients with $|k| \leqslant i$ and $|m| \leqslant j$ and, moreover, $k$ and $m$ must be of the same parity as $i$ and $j$, respectively. Due to the symmetries of the sinus and cosinus, we can assume that $k \geqslant 0$ and when $k=0$ we can assume $m \geqslant 0$. Finally, in the expansion of the frequency $\omega$ only terms with both $i$ and $j$ even appear.

Denote by $M, N, P$ the right-hand side terms of the first, second and third Equation in (5), respectively, and assume that $x, y, z$ have been obtained to order $n-1$, so the series $M, N$ and $P$ are known up to order $n$. Some terms of the left-hand side of (5) are also known: for instance, writing $x=x_{1}+x_{2}+\cdots+x_{n-1}$ and $\omega=1+\omega_{2}+\omega_{4}+\cdots+\omega_{n-2}$, where now the subindex denotes the order $(i+j)$ of the term, then in

$$
\dot{x}=\omega \frac{\partial x}{\partial \theta_{1}}+\omega \frac{\partial x}{\partial \theta_{2}},
$$


there are known $n$-order terms such as

$$
\omega_{2}\left(\frac{\partial x_{n-2}}{\partial \theta_{1}}+\frac{\partial x_{n-2}}{\partial \theta_{2}}\right)+\omega_{4}\left(\frac{\partial x_{n-4}}{\partial \theta_{1}}+\frac{\partial x_{n-4}}{\partial \theta_{2}}\right)+\cdots .
$$

Transferring to the right-hand side of (5) the known terms of order $n$, computed when $x, y, z$ are available at order $n-1$, we get new right-hand side terms, whose components at order $n$ are denoted by $\bar{M}_{n}, \bar{N}_{n}, \bar{P}_{n}$. In this way we have

$$
\begin{gathered}
\frac{\partial^{2} x_{n}}{\partial \theta_{1}^{2}}+2 \frac{\partial^{2} x_{n}}{\partial \theta_{1} \partial \theta_{2}}+\frac{\partial^{2} x_{n}}{\partial \theta_{2}^{2}}-2\left(\frac{\partial y_{n}}{\partial \theta_{1}}+\frac{\partial y_{n}}{\partial \theta_{2}}\right) \\
-3 x_{n}+\delta_{n} 2 \omega_{n-1}\left(\frac{\partial^{2} x_{1}}{\partial \theta_{1}^{2}}-\frac{\partial y_{1}}{\partial \theta_{1}}\right)=\bar{M}_{n}, \\
\frac{\partial^{2} y_{n}}{\partial \theta_{1}^{2}}+2 \frac{\partial^{2} y_{n}}{\partial \theta_{1} \partial \theta_{2}}+\frac{\partial^{2} y_{n}}{\partial \theta_{2}^{2}}+2\left(\frac{\partial x_{n}}{\partial \theta_{1}}+\frac{\partial x_{n}}{\partial \theta_{2}}\right) \\
+\delta_{n} 2 \omega_{n-1}\left(\frac{\partial^{2} y_{1}}{\partial \theta_{1}^{2}}+\frac{\partial x_{1}}{\partial \theta_{1}}\right)=\bar{N}_{n}, \\
\frac{\partial^{2} z_{n}}{\partial \theta_{1}^{2}}+2 \frac{\partial^{2} z_{n}}{\partial \theta_{1} \partial \theta_{2}}+\frac{\partial^{2} z_{n}}{\partial \theta_{2}^{2}} \\
\quad+z_{n}+\delta_{n} 2 \omega_{n-1} \frac{\partial^{2} z_{1}}{\partial \theta_{1}^{2}}=\bar{P}_{n},
\end{gathered}
$$

with $\delta_{n}=0$ if $n$ is even and $\delta_{n}=1$ if $n$ is odd. So, the linear system of equations for the unknown terms of order $n$ can be written as

$$
\begin{aligned}
-\left(3+(k+m)^{2}\right) x_{i j k m}-2(k+m) y_{i j k m}+2 \omega_{i-1, j} \delta_{1 k} \delta_{0 m} & =\bar{m}_{i j k m}, \\
-(k+m)^{2} y_{i j k m}-2(k+m) x_{i j k m}+2 \omega_{i-1, j} \delta_{1 k} \delta_{0 m} & =\bar{n}_{i j k m}, \\
\left(1-(k+m)^{2}\right) z_{i j k m}-2 \omega_{i, j-1} \delta_{0 k} \delta_{1 m} & =\bar{p}_{i j k m},
\end{aligned}
$$

where $\delta_{i j}$ stands for the Kronecker symbol and $\bar{m}_{i j k m}, \bar{n}_{i j k m}, \bar{p}_{i j k m}$ are the (known) coefficients of $\bar{M}_{n}, \bar{N}_{n}$ and $\bar{P}_{n}$, respectively.

To solve the above system we start with the last equation, for which we must consider the following two situations:

- If $(k, m)=(0,1)$ the coefficient in $z_{i j 01}$ is zero and we can solve for $\omega_{i, j-1}$. In this way we can compute all the $\omega_{i j}$ (recall that $i, j$ must be even). For these values of $k$ and $m$ we set $z_{i j 01}=0$.

- If $(k, m) \neq(0,1)$ the coefficient of $\omega_{n}$ is zero. In this situation the equation is solved for $z_{i j k m}$ except if $|k+m|=1$. For this last situation we also set $z_{i j k m}=0$. 
The determination of $x_{i j k m}$ and $y_{i j k m}$ is done as follows. Since all the $\omega_{n}$ are known after solving the last equation, the system to be considered is

$$
\begin{aligned}
-\left(3+(k+m)^{2}\right) x_{i j k m}-2(k+m) y_{i j k m} & =\bar{m}_{i j k m}-2 w_{i-1, j} \delta_{1 k} \delta_{0 m}, \\
-2(k+m) x_{i j k m}-(k+m)^{2} y_{i j k m} & =\bar{n}_{i j k m}-2 w_{i-1, j} \delta_{1 k} \delta_{0 m} .
\end{aligned}
$$

The determinant of this reduced linear system is

$$
\left((k+m)^{2}-1\right)(k+m)^{2},
$$

so we can solve (7) for $x_{i j k m}$ and $y_{i j k m}$ except if $k+m=0$ or $|k+m|=1$.

- When $k+m=0$, we normalise taking $y_{i j k m}=0$ and solve the first equation for $x_{i j k m}$.

- When $k+m=-1$, we normalise taking $x_{i j k m}=0$ and solve for $y_{i j k m}$.

- When $k+m=1$ :

- If $(k, m)=(0,1)$, we take $x_{i j 01}=y_{i j 01}=0$.

- In any other case, we normalise the solution taking $x_{i j 10}=0$ and solve the first equation for $y_{i j 10}$.

The implementation of the above procedure allows a fast computation of the asymptotic Lindstedt-Poincaré series (less than 5 seconds with an Intel Mobile Centrino processor at $1.7 \mathrm{GHz}$, when the expansions are computed up to order 25). Once the series are available, the computation of a particular orbit for given values of the amplitudes $\alpha$ and $\beta$ is a trivial fact. In Section 5 , we will discuss the numerical results obtained.

\section{A Second Procedure for the Determination of the Lindstedt-Poincaré Series}

In order to avoid the recurrences required for the evaluation of the righthand side of the equations of motion, one can introduce an additional variable $s$ through

$$
1+s=\frac{1}{\sqrt{(x+1)^{2}+y^{2}+z^{2}}} .
$$

This equation can also be written (gathering the different terms by orders) as 


$$
\begin{aligned}
& \overbrace{2(s+x)}^{\text {linear }}+\overbrace{x^{2}+y^{2}+z^{2}+s^{2}+4 x s}^{\text {quadratic }} \\
& \quad+\underbrace{2\left(x^{2}+y^{2}+z^{2}\right) s+2 x s^{2}}_{\text {cubic }}+\underbrace{\left(x^{2}+y^{2}+z^{2}\right) s^{2}}_{\text {quartic }}=0 .
\end{aligned}
$$

Using the new variable $s$, the equations of motion become

$$
\begin{aligned}
\ddot{x}-2 \dot{y}+3 s & =m, \\
\ddot{y}+2 \dot{x} & =n, \\
\ddot{z} & =p,
\end{aligned}
$$

to which we must add the algebraic relation (8). The right-hand side terms, $m, n$ and $p$, of (9) are polynomials in $x, y, z$ and $s$ of degree at least 2. Now, we look for the solution, at the different orders, of the system of Equations (8) and (9).

- From the linear terms of Equation (8), we find that

$$
s_{1}=-x_{1} .
$$

Substituting this value in (9) and retaining only the linear terms, we get

$$
\begin{aligned}
\ddot{x}_{1}-2 \dot{y}_{1}-3 x_{1} & =0, \\
\ddot{y}_{1}+2 \dot{x}_{1} & =0, \\
\ddot{z}_{1} & =0,
\end{aligned}
$$

whose solution is

$$
\begin{aligned}
& x_{1}=\alpha \cos \left(t+\phi_{1}\right)=-s_{1}, \\
& y_{1}=-2 \alpha \sin \left(t+\phi_{1}\right), \\
& z_{1}=\beta \cos \left(t+\phi_{2}\right) .
\end{aligned}
$$

- Once the linear part is solved, at each step we must solve for the unknown part of order $n$. Proceeding in an analogous way as in the previous section we get the linear system of equations,

$$
\begin{aligned}
-(k+m)^{2} x_{i j k m}-2(k+m) y_{i j k m}+3 s_{i j k m}+2 \omega_{i-1, j} \delta_{1 k} \delta_{0 m} & =\bar{m}_{i j k m}, \\
-(k+m)^{2} y_{i j k m}-2(k+m) x_{i j k m}+2 \omega_{i-1, j} \delta_{1 k} \delta_{0 m} & =\bar{n}_{i j k m}, \\
\left(1-(k+m)^{2}\right) z_{i j k m}-2 \omega_{i, j-1} \delta_{0 k} \delta_{1 m} & =\bar{p}_{i j k m}, \\
x_{i j k m}+s_{i j k m} & =q_{i j k m},
\end{aligned}
$$

where $\bar{m}_{i j k m}, \bar{n}_{i j k m}, \bar{p}_{i j k m}, q_{i j k m}$ are the (known) terms of the system. 
Combining the first equation of (10) with the last one, the system to be solved for $x_{i j k m}, y_{i j k m}, z_{i j k m}$ and $\omega$ becomes

$$
\begin{aligned}
-\left(3+(k+m)^{2}\right) x_{i j k m}-2(k+m) y_{i j k m}+2 \omega_{i-1, j} \delta_{1 k} \delta_{0 m} & =\bar{m}_{i j k m}-3 q_{i j k m}, \\
-(k+m)^{2} y_{i j k m}-2(k+m) x_{i j k m}+2 \omega_{i-1, j} \delta_{1 k} \delta_{0 m} & =\bar{n}_{i j k m}, \\
\left(1-(k+m)^{2}\right) z_{i j k m}-2 \omega_{i, j-1} \delta_{0 k} \delta_{1 m} & =\bar{p}_{i j k m} .
\end{aligned}
$$

Note that the left-hand side of these system is the same as in the previous section so the discussion about how to solve it follows in the same way. Once system (11) is solved we can set

$$
s_{i j k m}=-x_{i j k m}+q_{i j k m} .
$$

Of course, the values of the coefficients for the $x, y$ and $z$ series obtained with any of the two procedures described are identical, so there is no convergence improvement for any of both. The second method is much more faster than the first one, since all the recurrences for the Legendre functions are avoided.

\section{Numerical Results}

Table I shows the nonzero numerical values obtained for the coefficients of the Lindstedt-Poincaré series of the coordinates up to the fourth order. ${ }^{1}$ Of course, up to the third-order, these values agree with the ones computed by Richardson and Mitchell (2003).

It has been found that all the coefficients of the frequency series $\left(\omega_{20}\right.$, $\omega_{02}, \omega_{40}, \omega_{22}$ and $\left.\omega_{04}\right)$ are zero. The same result has been obtained for all the orders up to the maximum one explored $(N=i+j=35)$. This means that the orbits of the two-parametric family obtained are $2 \pi$-periodic and, as a consequence, the orbits of the leader and the follower have the same semi-major axis. We have not been able to prove that $\omega_{i j}=0$ for all $i, j$ with $i+j \geqslant 2$.

Some computations have been done to test the convergence of the formal series solutions. Essentially, the tests consist in comparing the numerically integrated trajectories against the series expansions evaluated during a certain time span, varying the values of the amplitudes $\alpha$ and $\beta$ and the phases $\phi_{1}$ and $\phi_{2}$. For fixed values of $\alpha$ and $\beta$, the convergence results are almost

${ }^{1}$ A file with the full set of coefficients up to a higher order can be obtained from the authors. 
TABLE I

Values of the coefficients of the Lindstedt-Poincare series of the coordinates, up to order four. The values that do not appear in the table are all equal to zero.

\begin{tabular}{|c|c|c|c|c|c|c|}
\hline$i$ & $j$ & $k$ & $m$ & $x_{i j k m}$ & $y_{i j k m}$ & $z_{i j k m}$ \\
\hline 1 & 0 & 1 & 0 & 1.000000 & -2.000000 & \\
\hline 0 & 1 & 0 & 1 & & & 1.000000 \\
\hline 2 & 0 & 0 & 0 & -0.500000 & 0.000000 & \\
\hline 2 & 0 & 2 & 0 & 0.500000 & 0.250000 & \\
\hline 0 & 2 & 0 & 0 & -0.250000 & 0.000000 & \\
\hline 0 & 2 & 0 & 2 & -0.250000 & 0.250000 & \\
\hline 1 & 1 & 1 & -1 & & & 1.500000 \\
\hline 1 & 1 & 1 & 1 & & & -0.500000 \\
\hline 3 & 0 & 1 & 0 & 0.000000 & 1.125000 & \\
\hline 3 & 0 & 3 & 0 & -0.375000 & -0.291666 & \\
\hline 1 & 2 & 1 & -2 & 0.000000 & 0.375000 & \\
\hline 1 & 2 & 1 & 0 & 0.000000 & -0.000000 & \\
\hline 1 & 2 & 1 & 2 & 0.125000 & -0.125000 & \\
\hline 2 & 1 & 0 & 1 & & & 0.000000 \\
\hline 2 & 1 & 2 & -1 & & & 0.000000 \\
\hline 2 & 1 & 2 & 1 & & & 0.375000 \\
\hline 0 & 3 & 0 & 1 & & & 0.000000 \\
\hline 0 & 3 & 0 & 3 & & & -0.000000 \\
\hline 4 & 0 & 0 & 0 & 0.359375 & 0.000000 & \\
\hline 4 & 0 & 2 & 0 & -0.708333 & -0.604166 & \\
\hline 4 & 0 & 4 & 0 & 0.348958 & 0.302083 & \\
\hline 2 & 2 & 0 & 0 & -0.374999 & 0.000000 & \\
\hline 2 & 2 & 0 & 2 & 0.250000 & 0.3 .1250 & \\
\hline 2 & 2 & 2 & -2 & -0.343750 & 0.000000 & \\
\hline 2 & 2 & 2 & 0 & 0.187500 & 0.000000 & \\
\hline 2 & 2 & 2 & 2 & -0.093749 & 0.093749 & \\
\hline 0 & 4 & 0 & 0 & -0.062500 & 0.000000 & \\
\hline 0 & 4 & 0 & 2 & -0.062500 & 0.062500 & \\
\hline 0 & 4 & 0 & 4 & 0.000000 & 0.000000 & \\
\hline 3 & 1 & 1 & -1 & & & 0.000000 \\
\hline 3 & 1 & 1 & 1 & & & 0.312500 \\
\hline 3 & 1 & 3 & -1 & & & 0.020833 \\
\hline 3 & 1 & 3 & 1 & & & -0.333333 \\
\hline 1 & 3 & 1 & -3 & & & -0.187499 \\
\hline 1 & 3 & 1 & -1 & & & 0.937499 \\
\hline 1 & 3 & 1 & 1 & & & -0.124999 \\
\hline 1 & 3 & 1 & 3 & & & 0.000000 \\
\hline
\end{tabular}


the same for any values of the phases, so here we will show only those corresponding to $\phi_{1}=\phi_{2}=0$. In Figure 2 we give the results obtained for the maximum difference between the numerical integration and the values obtained using the expansions for $t \in[0,2 \pi]$. This error is displayed as a function of the $\alpha$ and $\beta$ amplitudes. In the figure we display the results for both the order 5 and 25 solutions. Clearly, there is a great improvement in the convergence region of the order 25 solution in front of the order 5.

In Table II we display, for different values of $\alpha$, the maximum value of $\beta$ that can be reached for a given maximum threshold for the difference between the analytical solution of order 25 and the numerical integrated trajectory for $t \in[0,2 \pi]$. The numerical integration was done using a Runge-Kutta-Fehlberg 7-8 method with a bound for the local truncation
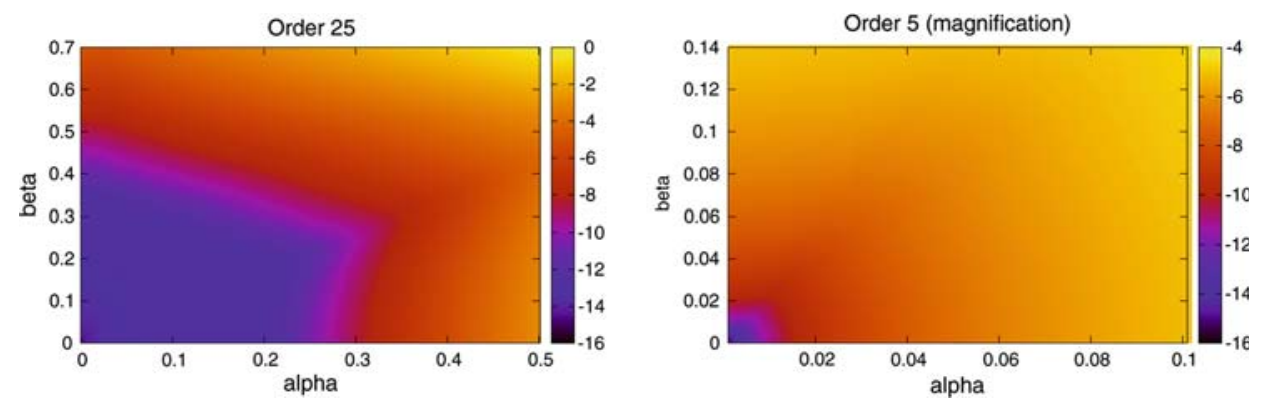

Figure 2. Convergence test results in the $\alpha-\beta$ plane for the orders 25 (left) and five expansions (right).

\section{TABLE II}

For several values of $\alpha$, the different columns display the maximum value of $\beta$ for which the difference between the order 25 analytical solution and the numerical integrated trajectory is less than a given threshold (displayed in the first row of the table).

\begin{tabular}{llllllllll}
\hline$\alpha$ & $10^{-5}$ & $10^{-6}$ & $10^{-7}$ & $10^{-8}$ & $10^{-9}$ & $10^{-10}$ & $10^{-11}$ & $10^{-12}$ & $10^{-13}$ \\
\hline 0.00 & 0.691 & 0.633 & 0.580 & 0.532 & 0.487 & 0.446 & 0.408 & 0.374 & 0.340 \\
0.05 & 0.682 & 0.621 & 0.564 & 0.512 & 0.464 & 0.421 & 0.382 & 0.347 & 0.297 \\
0.10 & 0.640 & 0.580 & 0.526 & 0.476 & 0.430 & 0.389 & 0.351 & 0.317 & 0.257 \\
0.15 & 0.604 & 0.546 & 0.493 & 0.444 & 0.400 & 0.359 & 0.318 & 0.279 & 0.207 \\
0.20 & 0.572 & 0.516 & 0.464 & 0.415 & 0.366 & 0.321 & 0.279 & 0.241 & 0.158 \\
0.25 & 0.544 & 0.488 & 0.432 & 0.378 & 0.329 & 0.284 & 0.245 & - & - \\
0.30 & 0.517 & 0.456 & 0.396 & 0.342 & 0.294 & - & - & - & - \\
0.35 & 0.487 & 0.421 & 0.361 & 0.302 & - & - & - & - & - \\
0.40 & 0.453 & 0.387 & - & - & - & - & - & - & - \\
0.45 & 0.418 & - & - & - & - & - & - & - & - \\
\hline
\end{tabular}


error, at each integration step, equal to $10^{-14}$. As it can be seen from the table (and also from Figure 2), there is a large domain of practical convergence, in the $\alpha-\beta$ plane, in which the expansions can be used instead of the numerical integration without any significant loss of precision.

The main advantage of the use of the expansions is that, once the coefficients of $x, y$ and $z$ have been obtained, they provide in a closed form the full family of bounded orbits. Any point of an orbit is computed just fixing the values that identify the orbit within the family (the amplitudes $\alpha$ and $\beta$ and the phases $\phi_{1}, \phi_{2}$ ) and the epoch $t$ at which we want to evaluate the solution.

Figures 3 to 6 show the orbits computed for different values of the amplitudes and the phases. For these representations we have used both the inertial reference system, in which the orbits are elliptic Keplerian orbits,
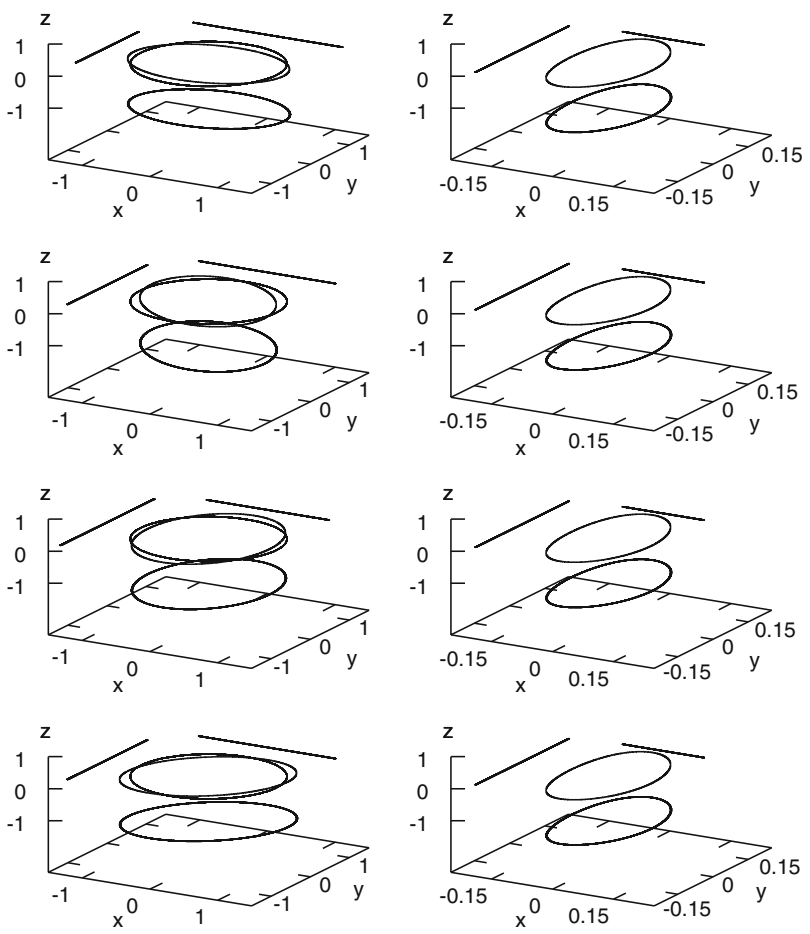

Figure 3. From top to bottom, 3D representation and coordinate planes projections of orbits with the same amplitudes, $\alpha=0.1, \beta=0$, and different phases: $\left(\phi_{1}, \phi_{2}\right)=(0,0),(\pi / 2,0),(\pi, 0)$ and $((3 / 4) \pi, 0)$. The orbits on the left column are represented in the inertial reference frame and the ones on the right column in the Hill's reference frame. In the inertial frame representations, the circular reference orbit of radius $r_{l}=1$ is also displayed. As is clearly seen in the projections, the orbit of the follower is coplanar with the one of the leader, since $\beta=0$ in all the cases. 

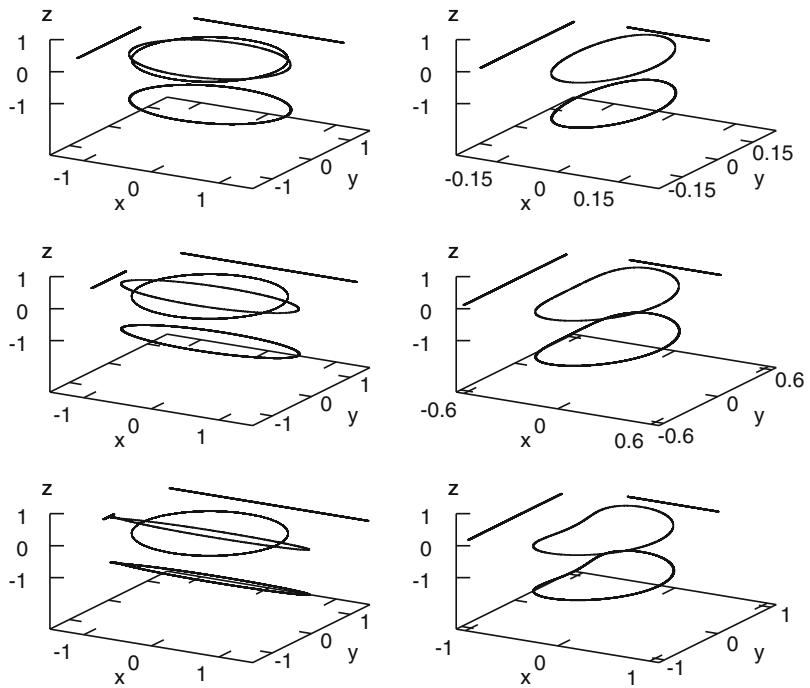

Figure 4. From top to bottom, 3D representation and coordinate planes projections of orbits with $(\alpha, \beta)=(0.1,0),(0.3,0),(0.5,0)$ and $\left(\phi_{1}, \phi_{2}\right)=(0,0)$. The orbits on the left column are represented in the inertial reference frame and the ones on the right column in the Hill's reference frame. In the inertial frame representations, the circular reference orbit of radius $r_{l}=1$ is also displayed. Note that the orbit with $(\alpha, \beta)=(0.5,0)$ is not in the convergence domain of the expansions, but is rather close to a Keplerian orbit.
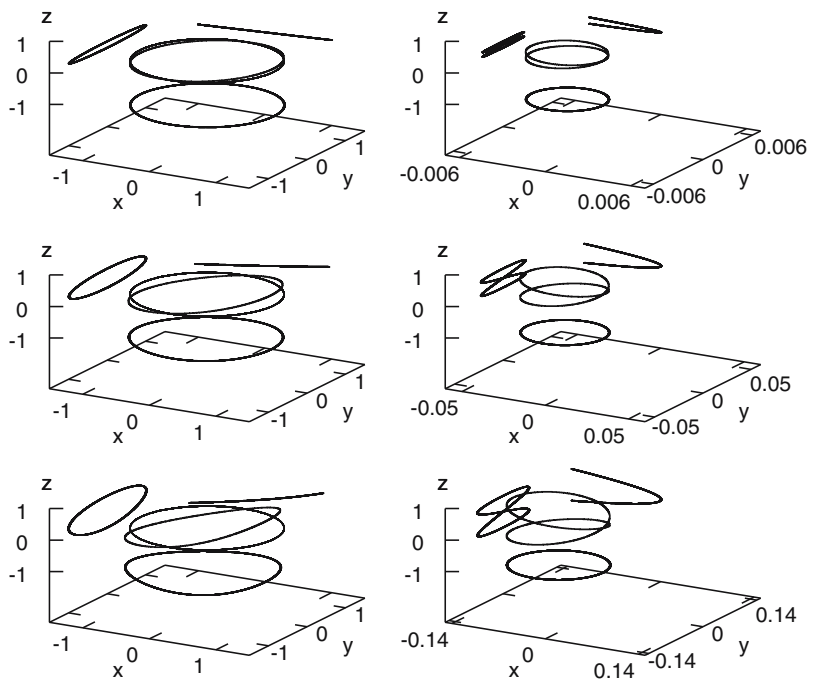

Figure 5. From top to bottom, 3D representation and coordinate planes projections of orbits with $(\alpha, \beta)=(0.0,0.1),(0.0,0.3),(0.0,0.5)$ and $\left(\phi_{1}, \phi_{2}\right)=(0,0)$. The orbits on the left column are represented in the inertial reference frame and the ones on the right column in the Hill's reference frame. In the inertial frame representations, the circular reference orbit of radius $r_{l}=1$ is also displayed. 

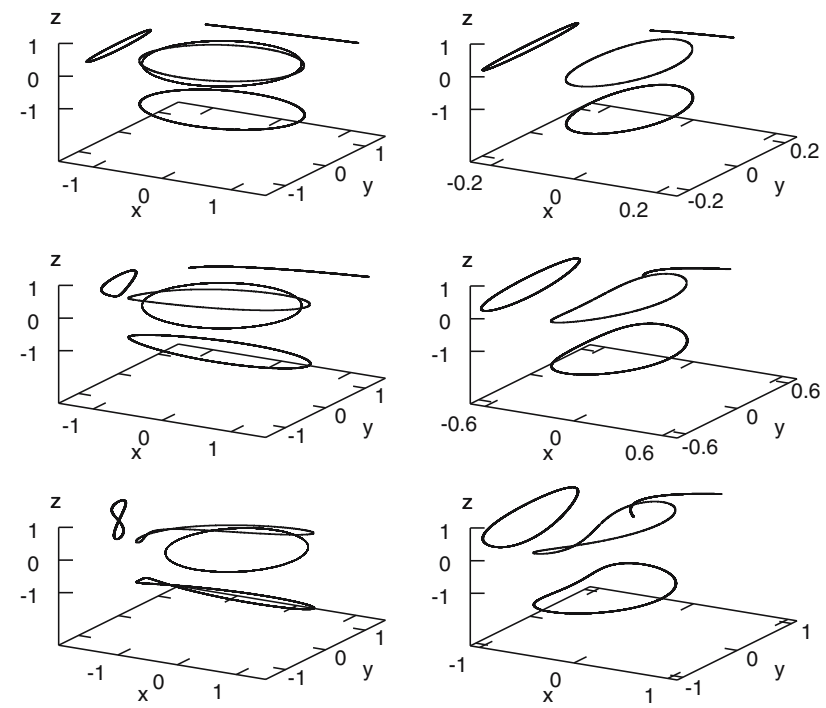

Figure 6. From top to bottom and left to right, 3D representation and coordinate planes projections of orbits with $(\alpha, \beta)=(0.1,0.1),(0.3,0.3),(0.5,0.5)$ and $\left(\phi_{1}, \phi_{2}\right)=(0,0)$. The circular reference orbit of radius $r_{l}=1$ is also displayed. The orbits on the left column are represented in the inertial reference frame and the ones on the right column in the Hill's reference frame. Clearly, the orbit computed for $(\alpha, \beta)=(0.5,0.5)$ is not a Keplerian orbit, since for these values of the amplitudes the expansions are far form the practical convergence region.

and Hill's reference frame, which is the system where the orbits have been computed. Of course, the expansions can be evaluated for values of the amplitudes very far from the practical convergence region (see Figure 6), in this case the shape of the orbit is far from a Keplerian one.

\section{Acknowledgements}

This work has been been partially supported by grants CIRIT 2001SGR70, 2003XT-00021 (Catalonia) and grant BFM2003-09504 (MCYT, Spain). M. Marcote acknowledges the support of the doctoral research grant AP2001-3064 (MECD, Spain). 


\section{References}

Abramowitz, M. and Stegun, I.A.: 1972, Handbook of Mathematical Functions, Dover Publications, Inc, NewYork.

Gómez, G., Jorba, A., Masdemont, J.J. and Simó, C.: 2001, Dynamics and Mission Design Near Libration Point Orbits - Volume 3: Advanced Methods for Collinear Points, World Scientific, Singapore.

Masdemont, J.J.: 2005, 'High Order Expansions of Invariant manifolds of Libration Point Orbits with Applications to Mission Design', Dyn. Syst. an Int. J. 20, 59-113.

Richardson, D.L.: 1980, 'A Note on a Lagrangian Formulation for Motion about the Collinear Points', Cel. Mech. 22, 231-235.

Richardson, D.L. and Mitchell, J.W.: 2003, 'A third-Order Analytical Solution for Relative Motion with a Circular Reference Orbit', J. Astron. Sci. 51, 1-12. 